Gerión. Revista de Historia Antigua

ISSN: 0213-0181

http://dx.doi.org/10.5209/geri.68595

\title{
Y de una inscripción nacieron siete: la suerte de una placa funeraria de Gades $^{1}$
}

\author{
Ricardo de Balbín-Bueno - Helena Gimeno Pascual - Helena Lorenzo Ferragut ${ }^{2}$
}

Recibido: 10 de enero de 2019 / Aceptado: 29 de mayo de 2019

Resumen: Presentamos un epígrafe de Gades conservado en el Museo de Cádiz considerado desaparecido poco después de su hallazgo, cuyos diferentes fragmentos fueron publicados por diversos autores como si de inscripciones independientes se tratasen.

Palabras clave: Epigrafía; Gades; Cádiz; conventus Gaditanus; Historiografía.

\section{[en] Seven Inscriptions that came from One: the Fate of a Funerary Plaque from Gades}

Abstract: We present an epigraph from Gades held at Museo de Cádiz. It was considered lost shortly after its discovery and its different parts were published by various scholars as diverse independent inscriptions, without noticing they belonged to the same epigraph.

Keywords: Epigraphy; Gades; Cádiz; Conventus Gaditanus; Historiography.

Sumario. 1. Introducción. 2. Descripción de la inscripción en su estado actual. 3. Historia de la transmisión. 4. Comentario. 5. Referencias bibliográficas.

Cómo citar: Balbín-Bueno, R. de - Gimeno Pascual, H. - Lorenzo Ferragut, H. (2020): Y de una inscripción nacieron siete: la suerte de una placa funeraria de Gades, Gerión 38/1, 295-305.

\footnotetext{
$1 \quad$ Este trabajo se ha realizado en el marco del proyecto: "Nueva edición de CIL II: - 1. Inscripciones del extremo occidental del conventus Gaditanus (CIL II ${ }^{2} / 6$ ), FFI2016-77528-P (MINECO/FEDER)”. Queremos dejar constancia de nuestro profundo agradecimiento al Museo de Cádiz y en particular a Ma Dolores López de la Orden por su inestimable ayuda y colaboración.

2 Centro CIL II. Universidad de Alcalá de Henares.

E-mail: ricardo.balbin@uah.es; helena.gimeno@uah.es; helena.lorenzo@uah.es
} 


\section{Introducción}

El proyecto de investigación dedicado a la epigrafía del extremo occidental del conventus Gaditanus, en el ámbito de la nueva edición del Corpus Inscriptionum Latinarum (CIL $\left.\mathrm{II}^{2}\right)$, ha incluido una campaña de documentación en el Museo de Cádiz en el año 2018, en la que hemos verificado que una inscripción, hoy rota en tres fragmentos, ha sido publicada como siete inscripciones diferentes.

Gracias a la autopsia y al estudio bibliográfico de las ediciones de la pieza, hemos podido subsanar errores anteriores de transcripción. Ya la primera publicación, en 1928, por su descubridor Pelayo Quintero Atauri, provoca dudas ante la falta de precisión a la hora de transmitir el texto. Destaca la carencia de signos diacríticos epigráficos específicos, tónica habitual en sus publicaciones, ya que este autor fue un arqueólogo más centrado en los contextos de hallazgo que en el estudio de las piezas epigráficas.

El registro que se conserva de estas intervenciones ha de ser estudiado con suma cautela, y hay que recurrir a los años anteriores o posteriores a los hallazgos para obtener una información más fidedigna ya que, a veces, las referencias topográficas que da el autor se refieren a puntos que ha publicado en memorias anteriores. Este es el caso de las excavaciones de 1928, que están estrechamente relacionadas con las de 1927, momento en el que Quintero comienza a descubrir recintos funerarios colectivos en Cádiz, junto a la muralla moderna de la ciudad, que había empezado a excavar en $1926^{3}$ y -por el mapa que añade en este año a su memoria- lo hace a la altura de Puerta de Tierra, junto a la orilla atlántica. Es en las memorias de 1927 donde describe el modelo que encuentra de recinto colectivo, ${ }^{4}$ documentando hasta tres de este tipo en dicha campaña y lo que podrían ser los cimientos de otros tantos, motivo por el que decide excavar en esa misma zona al año siguiente. ${ }^{5}$ Esta es la campaña en la que se descubre el epígrafe que nos ocupa, aunque dada la complejidad de las memorias de Quintero, no es extraño que se haya publicado también como hallado en 1927, al menos uno de sus fragmentos. ${ }^{6}$ En esta misma publicación se especifica que aparece dentro del columbario de la familia Argentilia, dato que no aparece ni en las memorias de Quintero ni en las sucesivas publicaciones, hasta la mencionada. En sus memorias Quintero no especifica a qué columbario pudo pertenecer tanto esta placa, como tampoco explica con cuál se relacionaban las restantes halladas en la misma campaña, ${ }^{7}$ dedicadas a Chrescens $^{8}$ (inv. $\mathrm{n}^{\circ}$ 4619), L. Licinius Rufus $^{9}$ (inv. ${ }^{\circ}$ 4618), Ausesis $^{10}$ (inv. $\mathrm{n}^{\mathrm{o}} 6757$ ) y [- - - erius $^{11}$ (inv. $\mathrm{n}^{\mathrm{o}} 4631$ ).

Al mencionado arqueólogo le debemos el descubrimiento de amplias zonas de enterramiento, ejemplo que siguen las intervenciones de Francisco Cervera ${ }^{12}$ y M $^{\mathrm{a}}$

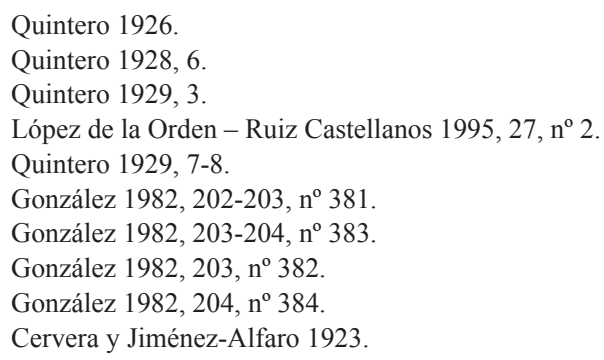


Josefa Jiménez Cisneros ${ }^{13}$ hasta los años cincuenta del siglo XX. A partir de este momento las excavaciones pasan a depender casi exclusivamente de intervenciones de urgencia relacionadas con procesos urbanísticos. Estas aportan en mayor o menor medida información sobre la parcela a la que afectan, sin interpretar los hallazgos introduciéndolos en el conjunto gaditano. Los trabajos que se han dedicado a tratar la necrópolis romana y sus dinámicas ${ }^{14}$ no son suficientes ni siquiera para acercarnos a comprender este conjunto funerario en su organización básica. El principal problema es la antigüedad de ambos trabajos y la ingente cantidad de informes arqueológicos a consultar para disponer de una base de estudio fiable. Sin un estudio completo y actualizado de los datos arqueológicos del contexto funerario romano en Cádiz, podemos únicamente dar cuenta de la diversidad y aparente "caos" que existe en la gran necrópolis gaditana. La zona más estudiada, y por tanto con mayor número de hallazgos, comienza a partir de Puerta Tierra, límite de época moderna de la ciudad. También existen no pocos hallazgos hacia el interior de la ciudad moderna, ${ }^{15}$ pero son escasamente representativos respecto al total. La zona existente inmediatamente a continuación de Puerta Tierra fue excavada en amplitud por Quintero, y en sus aledaños documenta una gran cantidad de enterramientos y recintos funerarios de diversas épocas. Destaca la abundancia de recintos funerarios colectivos, quizás una de las causas de que el registro epigráfico gaditano se componga casi por entero de placas. Estas también han aparecido encastradas en estelas de piedra ostionera, de muy diferente tipología. ${ }^{16}$

Todo el registro funerario se ve afectado por la misma circunstancia: la rapidez y constancia de expolio y reutilización, sobre todo en época antigua. La interpretación o adscripción del material epigráfico a espacios específicos, incluso en las intervenciones más recientes, se hace tarea complicada, ya que es habitual que aparezca formando parte de tumbas más recientes, sin poder asegurar que es la primera vez que se reutiliza o el periodo de tiempo transcurrido desde su erección hasta su reutilización.

\section{Descripción de la inscripción en su estado actual}

Se trata de una placa de mármol (Fig. 1) a la que le falta la esquina superior izquierda. Está rota en tres partes que denominaremos $a, b$ y $c$, conservadas todas en el Museo de Cádiz.

La parte $a$, rota a la izquierda y por debajo, está partida en dos fragmentos que encajan y mide $13,5 \mathrm{~cm}$ de alto, 14,5 de ancho y 1,5 de grueso (inv. $\mathrm{n}^{\circ} 4633$ ).

La parte $b$, rota arriba y a la derecha, mide $13 \mathrm{~cm}$ de alto, 12 de ancho y 2 de grueso (inv. $\mathrm{n}^{\mathrm{o}}$ 11349).

La parte $c$, rota por arriba y a la izquierda, tiene en el margen inferior un agujero con restos de metal del perno de un clavo de fijación; mide $8,8 \mathrm{~cm}$ de alto, 7,8 cm de ancho y 2 de grueso (inv. $n^{\circ} 11357$ ).

\footnotetext{
Jiménez Cisneros 1971.

Corzo Sánchez 1992; Morales Muñoz 2006.

Morales Muñoz 2006, 34-35.

De Balbín-Bueno (e.p.), como caso específico.
} 


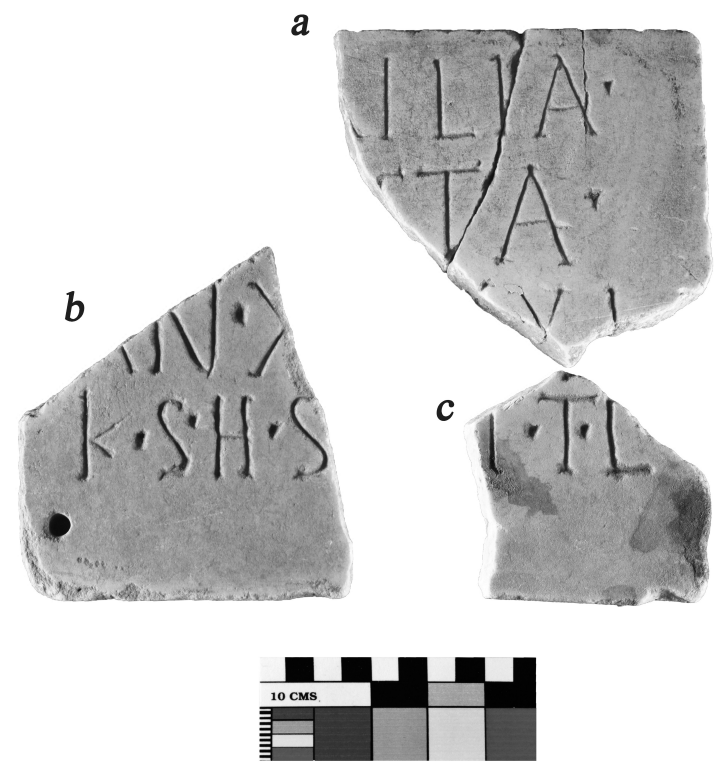

Fig. 1. Placa hallada en 1928 en Cádiz, en el mausoleo de la familia Argentilia, con referencia a los tres fragmentos que la conforman: $a, b$ y $c$ (Ricardo de Balbín-Bueno).

Las letras miden 3,2 cm de alto en la línea 1, 3,5 cm en las líneas 2-3 y $3 \mathrm{~cm}$ en la cuarta. El signo de interpunción es triangular. El texto dice:

$$
\begin{gathered}
{[\text { Ae]milia }} \\
{[---] \text { sta }} \\
\text { an }(\text { norum }) \cdot X[. ?] X X I \\
k(\text { ara }) \cdot s(\text { uis }) \cdot h(\text { ic }) \cdot s(\text { ita })[e(s t) \cdot s(\text { it })] t(\text { ibi }) \cdot t(\text { erra }) \cdot l(\text { evis })
\end{gathered}
$$

\section{Historia de la transmisión}

Publicada por primera vez en las Memorias de la Junta Superior de Excavaciones y Antigüedades del año 1928 por Quintero, ${ }^{17}$ indicaba este autor que estaba rota en tres partes que formaban un rectángulo de $23 \mathrm{~cm}$, pero descuidó señalar que los inicios de las dos primeras líneas eran suplementos suyos. En esta forma, como si estuviese completa, fue transmitida por Enrique Romero de Torres en su Catálogo Monumental ${ }^{18}$-quien, sin embargo, advirtió que era un triángulo-, por José Vives ${ }^{19}$ $\mathrm{y}$, en último lugar, por Julián González. ${ }^{20}$

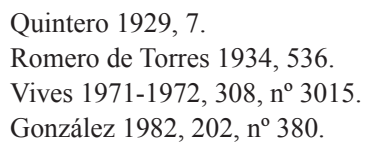




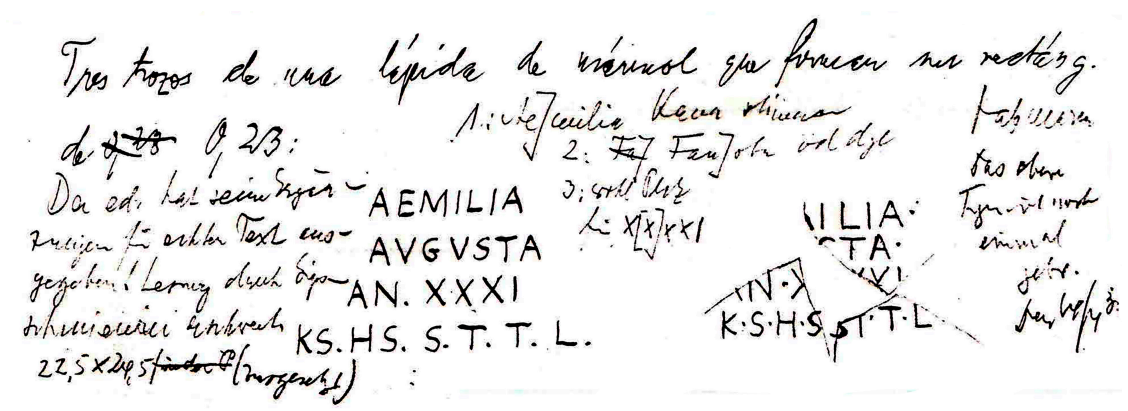

Fig. 2. Scheda de Lothar Wickert donde describe el estado en el que se encontraba la pieza cuando él la vio en 1931.

En 1931 Lothar Wickert, en su segundo viaje a España para la edición de un nuevo suplemento a $C I L \mathrm{II},{ }^{21}$ visitó Cádiz para verificar la edición de Quintero y, al ver la pieza, anotó en su scheda ${ }^{22}$ (Fig. 2) que éste había incluido sus suplementos. Indicó también que la parte $b$ estaba rota en dos: "das obere Fragment noch einmal gebrochen". Esta es la última noticia que tenemos de esta placa hasta la edición de González de las inscripciones romanas de la provincia de Cádiz de 1982 y, por cierto, la única que ofrece una descripción correcta de la inscripción. Por el camino las tres partes se dispersaron, incluidos los dos fragmentos que constituyen la parte $a$. El resultado fue que de una sola inscripción nacieron las siete siguientes:

1. La inscripción con los suplementos de Quintero fue dada por perdida, como ya hemos dicho, en la edición de González ${ }^{23}$ y publicada en la forma siguiente (Fig. 3):
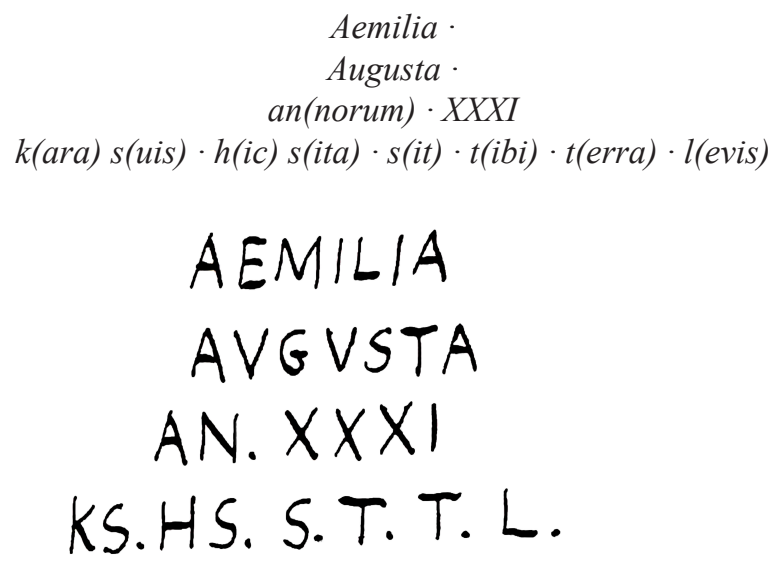

Fig. 3. Lectura dada por P. Quintero y transmitida por J. González, extraída de la scheda de Wickert.

$21 \quad$ Para los viajes de Wickert a España véase Rothenhöfer 2014.

22 Las schedae originales se conservan en la Academia de Berlín y existe una copia en el Centro CIL II de Alcalá de Henares.

23 González 1982, 202, nº 380. 
2-3. González publicó por separado los dos fragmentos $a .1$ (Fig. 4) y a.2 (Fig. 5) de la parte $a^{24}$ como si fueran dos inscripciones distintas:

a.1 González 1982, nº 476

ILI

ST a.2 González 1982, nº 493

A

A

XI

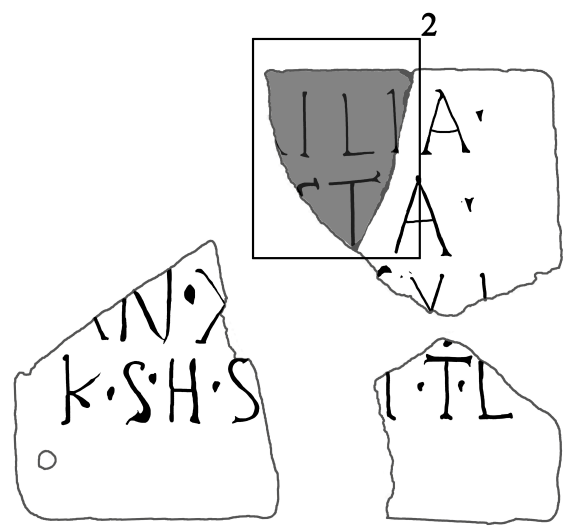

Fig. 4. Dibujo de la pieza con referencia al fragmento específico publicado por J. González 1982, no 476 (Ricardo de Balbín-Bueno).
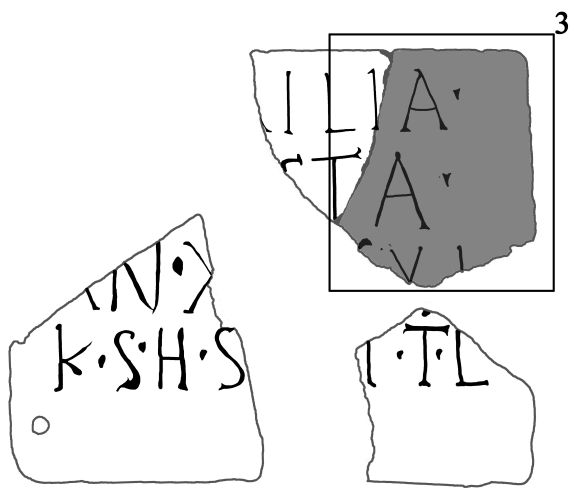

Fig. 5. Dibujo de la pieza con referencia al fragmento específico publicado por J. González 1982, no 493 (Ricardo de Balbín-Bueno).

4. Publicó también González la parte $b$ bajo un número diferente ${ }^{25}$ (Fig. 6):

$\mathrm{AN} \cdot \mathrm{X}$

$\mathrm{K} \cdot \mathrm{S} \cdot \mathrm{H} \cdot \mathrm{S} \cdot[\mathrm{E}]$ 


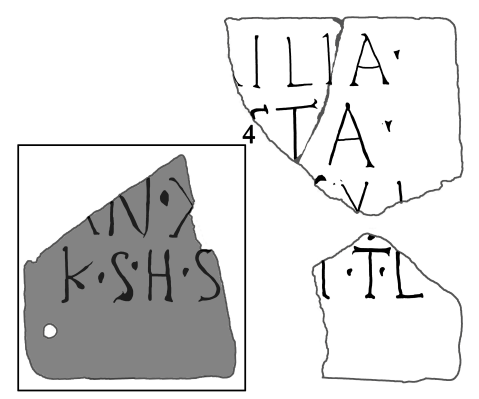

Fig. 6. Dibujo de la pieza con referencia al fragmento específico publicado por J. González 1982, no 494 (Ricardo de Balbín-Bueno).

5. En $1995 \mathrm{M}^{\mathrm{a}}$ Dolores López de la Orden y Antonio Ruiz Castellanos, en sus Nuevas inscripciones del Museo de Cádiz, ${ }^{26}$ editaron como inédita la parte $a$ (Fig. 7) -ahora con los dos fragmentos $a .1$ y a.2 unidos de nuevo- (inv. $\mathrm{n}^{\circ} 4633$ ) con el siguiente texto:

$$
\begin{aligned}
& {[- \text { - }] \text { milia }} \\
& \text { [-- - } \\
& \text { [- - - ]XXIa. }
\end{aligned}
$$

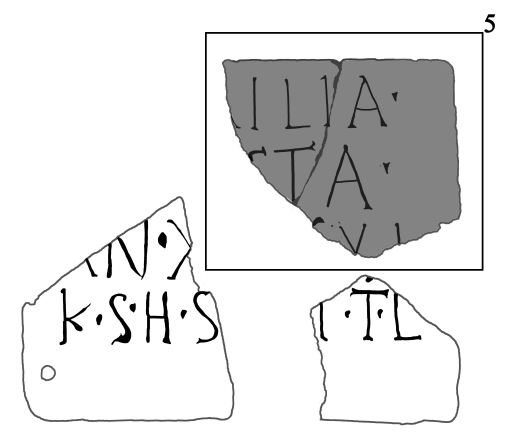

Fig. 7. Dibujo de la pieza con referencia al fragmento específico publicado por $\mathrm{M}^{\mathrm{a}} \mathrm{D}$. López de la Orden - A. Ruiz Castellanos 1995, nº 2 (Ricardo de Balbín-Bueno).

6. La parte $c$ (Fig. 8) fue publicada por González con foto, ${ }^{27}$ pero la identificó -mal- con otra inscripción hallada por Quintero en los Baños del Blanco en 1917 y publicada en las Memorias de la Junta Superior de Excavaciones, Antigüedades y Museos del año $1917 .{ }^{28}$ En esta última se leía T.L. y fue recogida también por Romero de Torres. ${ }^{29}$ González no indica ni lugar de hallazgo ni número de inventario del Museo y ofrece el texto siguiente:

López de la Orden - Ruiz Castellanos 1995, 27, nº 2, lám. 1 (=AE 1995, 778 =HEp 6, 1996, 255).

González 1982, 191-192, nº 357.

Quintero 1918, 5.

Romero de Torres 1934, 165, $\mathrm{n}^{\circ} 2$. 


$$
[--s(i t) t(\text { ibi) }] t(\text { erra }) \cdot l(\text { evis })
$$

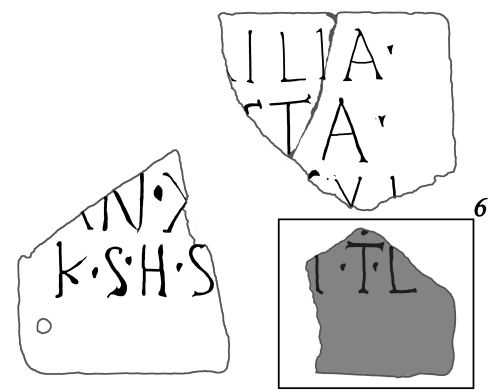

Fig. 8. Dibujo de la pieza con referencia al fragmento específico publicado por J. González 1982, no 357 (Ricardo de Balbín-Bueno).

No sería imposible que la que él mismo publica como inédita bajo su $\mathrm{n}^{\mathrm{o}} 465^{30}$ (inv. n. 4629) sea la encontrada por Quintero en los Baños del Blanco (Fig. 9), aunque en contra de ello estaría que en la ficha del museo consta que esta fue hallada en 1916 en Punta de Vaca en la Huerta de Villa Antonia.

357. Museo Arqueológico de Cádiz.

P. Quintero, Memorias 18 (1917); $\rightarrow$ Romero de Torres, CMCádiz, p. 164.

Fragmento de inscripción funeraria, de mármol blanco, tiene unas medidas máximas de $0,085 \mathrm{~m} . \times 0,08 \mathrm{~m} . \times 0,02 \mathrm{~m}$. Las letras son capitales cuadradas, de $2,5 \mathrm{~cm}$. de altura. Los puntos son triangulares, (cf. lám. LXXX):

\section{T . L}

$[S($ it) t(ibi)] t(erra) I(evis).

Esto es: ... Sea para ti la tierra leve.
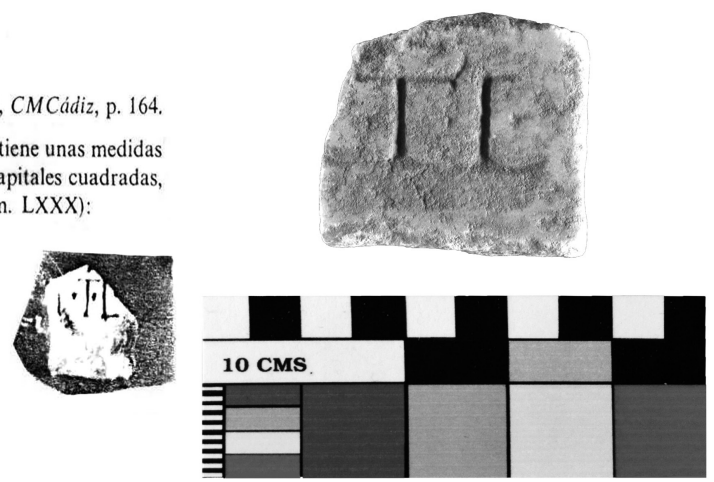

Fig. 9. A la izquierda: publicación de J. González (1982, no 357) de la parte $c$. A la derecha: fragmento de placa con $\mathrm{n}^{\mathrm{o}}$ de inventario 4629 hallado en Baños del Blanco en 1917

(Ricardo de Balbín-Bueno).

7. López de la Orden y Ruiz Castellanos ${ }^{31}$ publican esta parte $c$ (Fig. 10) (inv. $\mathrm{n}^{\mathbf{o}}$ 11357) -y de ellos la toma HEp 6, 1996, 280-como una inscripción inédita, con el texto siguiente:

$$
[--s(i t)] t(\text { ibi }) \cdot t(\text { erra }) \cdot l(\text { evis })
$$




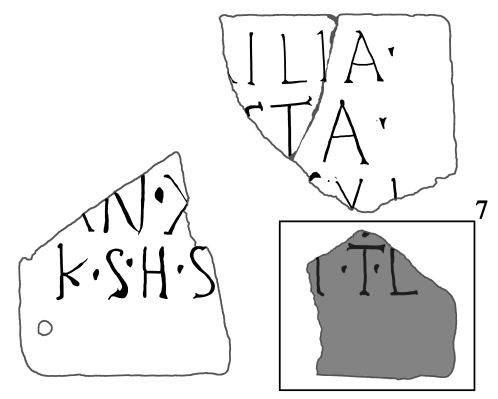

Fig. 10. Dibujo de la pieza con referencia al fragmento específico publicado por $\mathrm{M}^{\mathrm{a}} \mathrm{D}$. López de la Orden - A. Ruiz Castellanos 1995, nº 29 (Ricardo de Balbín-Bueno).

\begin{tabular}{|c|c|c|c|c|}
\hline & INSCRIPCIÓN 1 & \multicolumn{3}{|c|}{ INSCRIPCIONES 2-7 } \\
\hline & QUINTERO $1927^{1}$ & \multicolumn{2}{|c|}{$\begin{array}{l}\text { GONZÁLEZ 1982: } 476^{2}, 493^{3} \text {, } \\
494^{4}, 357^{6}\end{array}$} & $\begin{array}{l}\text { LÓPEZ DE LA ORDEN - RUIZ } \\
\text { CASTELLANOS 1995: } 2^{5}, 29^{7}\end{array}$ \\
\hline V. 1 & AEMILIA $^{1}$ & $\mathrm{ILI}^{2}$ & $\mathrm{~A}^{3}$ & [- - -]MILIA · 5 \\
\hline V. 2 & AVGVSTA $^{1}$ & $\mathrm{ST}^{2}$ & $\mathrm{~A}^{3}$ & {$[---]$ STA $\cdot 5$} \\
\hline V. 3 & AN XXXI ${ }^{1}$ & $\mathrm{AN} \cdot \mathrm{X}^{4}$ & $\mathrm{XI}^{3}$ & {$[---] X^{2} I^{5}$} \\
\hline V. 4 & KS HS STTL ${ }^{1}$ & $\mathrm{~K} \cdot \mathrm{S} \cdot \mathrm{H} \cdot \mathrm{S} \cdot[\mathrm{E}]^{4}$ & $\mathrm{~T} \cdot \mathrm{L}^{6}$ & {$[--\mathrm{S}] \mathrm{T} \cdot \mathrm{T} \cdot \mathrm{L}^{7}$} \\
\hline
\end{tabular}

Fig. 11. Tabla que desarrolla las diferentes publicaciones según qué partes del texto se publican. Los números en superíndice se refieren a nuestra numeración de 1 a 7 de las inscripciones generadas.

\section{Comentario}

La inscripción menciona el nomen [- - - milia y el cognomen [- - -]sta de la difunta. El nomen puede fácilmente reconstruirse como Aemilia, tal y como propuso P. Quintero; Aemilius, - $a$ es frecuente en Cádiz. ${ }^{32}$ Respecto al cognomen, Pelayo Quintero editó Augusta; sin embargo, la ausencia de signos diacríticos ya comentada nos impide determinar si el cognomen era realmente este o si se trata de una hipótesis aventurada por el arqueólogo.

Augustus, - $a$ es un cognomen muy poco común en la onomástica latina, por tanto, serían más probables otros como [Fau]sta o quizá [Iu]sta. En este segundo caso, incluso se podría aventurar una filiación o una indicación de patronazgo entre nomen

32 En Cádiz y su provincia hay 6 testimonios de este nomen (CIL II $1750=$ González 1982, 94-95, nº 146; HEp 6, 1996, 278; HEp 6, 1996, 526; CIL II 1751 = González 1982, 95, nº 147; CIL II 1752 = González 1982, 95-96, nº 148; CIL II 1753 = González 1982, 96, nº 149). 
y cognomen. Del cognomen Faustus, - a hay otros dos ejemplos en la ciudad. ${ }^{33}$

La inscripción es un ejemplar típico de la epigrafía funeraria gaditana: una placa de mármol con los datos onomásticos y biométricos, la fórmula laudatoria y las fórmulas de deposición. Para loar a la difunta se utiliza la expresión c(ara) s(uis) -aquí con el fonema $k$-de uso muy frecuente en la ciudad de Gades, en contraposición con la fórmula pius, - $a$ in suis, mucho más extendida en la Baetica.

En cuanto a la datación podría fecharse hacia mediados o finales del siglo II d.C., por el tipo de letra, las fórmulas y el uso de la letra $\mathrm{k}$.

Para concluir queremos resaltar que la autopsia de las inscripciones y el estudio de la transmisión de las mismas se revelan esenciales en casos como el que aquí analizamos, no sólo para la edición óptima del epígrafe, sino también para determinar el valor de sus editiones principes.

\section{Referencias bibliográficas}

Balbín-Bueno, R. de (e.p.): "Identity and Death in the necropolises of Cádiz (Spain) through their funerary monuments' biography. The case of Lucius Popillius Acastus", AEspA.

Cervera y Jiménez-Alfaro, F. (1923): Excavaciones en extramuros de Cádiz: Memoria acerca de los trabajos y resultados obtenidos en dichas excavaciones (=Junta Superior de Excavaciones y Antigüedades 57), Madrid.

Corzo Sánchez, R. (1992): “Topografía y ritual en la necrópolis de Cádiz”, Spal 1, 263-292 (http://dx.doi.org/10.12795/spal.1992.i1.13).

González, J. (1982): Inscripciones romanas de la provincia de Cádiz, Cádiz.

Jiménez Cisneros, Mª J. (1971): Historia de Cádiz en la antigüedad, Cádiz.

López de la Orden, Ma D. - Ruiz Castellanos, A. (1995): Nuevas inscripciones latinas del Museo de Cádiz, Cádiz.

Morales Muñoz, C. M. (2006): "La necrópolis de Gades: la complejidad de su ubicación”, Ubi Sunt? 20, 32-41.

Quintero, P.

(1918): "Excavaciones en extramuros de la ciudad de Cádiz: Memoria acerca de los resultados obtenidos en 1917", MJSEA 18 (1917/4).

(1926): "Memoria de las excavaciones practicadas en 1925-1926", MJSEA 84 (19251926/2).

(1928): "Excavaciones en extramuros de Cádiz: memoria de las excavaciones practicadas en 1927", MJSEA 95 (1927).

(1929): "Excavaciones de Cádiz: memoria de las excavaciones practicadas en 1928", MJSEA 99 (1928/1).

Romero de Torres, E. (1934): Catálogo Monumental de España. Provincia de Cádiz (19081909), Madrid.

Rothenhöfer, P. (2014): "Siguiendo los pasos de Emil Hübner. Lothar Wickert y su trabajo para un Suplementum Hispaniense del Corpus Inscriptionum Latinarum II", [en] D. Marzoli - J. Maier Allende - T. G. Schattner (coords.), Historia del Instituto Arqueológico Alemán de Madrid. Fascículo 4. Emil Hübner y las ciencias de la antigüedad clásica en Hispania, Actas del coloquio celebrado en Madrid del 19 al 20 de noviembre del 2008

33 González 1982, 181-182, n 337; González 1982, 216, nº 409. 
por el 175 aniversario del nacimiento de Emil Hübner (=Iberia Archaeologica 14/4), Darmstadt, 337-339.

Vives, J. (1971): Inscripciones latinas de la España romana, Barcelona. 